\title{
Principais recomendações em cuidados pré-operatórios
}

\section{Main recommendations in preoperative care}

Josiani dos Santos Garcez¹. Liane Carvalho de Brito de Sousa². Maria Barreto Novais Neta ${ }^{3}$. Flávio Lobo Maia $^{4}$. Fernanda Paula Cavalcante Araújo 5 .

1 Residência médica em anestesiologia, Hospital Universitário Walter Cantídio (HUWC), Fortaleza, Ceará, Brasil. 2 Médica anestesiologista, coordenadora do Serviço de cuidados paliativos do Hospital Universitário Walter Cantídio (HUWC), Fortaleza, Ceará, Brasil. 3 Médica anestesiologista, Preceptora da residência em anestesiologia, Hospital Universitário Walter Cantídio (HUWC), Fortaleza, Ceará, Brasil. 4 Médico anestesiologista, preceptor da residência em anestesiologia, Hospital Universitário Walter Cantídio (HUWC), Fortaleza, Ceará, Brasil. 5 Médica anestesiologista, Doutora em anestesiologia, Universidade de São Paulo (USP), São Paulo, São Paulo, Brasil.

\section{RESUMO}

Introdução: a avaliação pré-anestésica (APA) é uma prática imprescindível no cuidado perioperatório, por oferecer condições de planejamento do ato anestésico e cirúrgico, mantendo a segurança física e emocional do paciente. Objetivo: apresentar uma revisão das principais orientações pré-anestésicas disponíveis na literatura. Metodologia: trabalho de revisão bibliográfica realizada nos domínios PubMed, SciELO, British Journal of Anaesthesia (BJA), Aneshesiology e Current Opinion. Resultados: as principais recomendações abordam a estratificação do risco anestésico/cirúrgico, jejum pré-operatório, controle da ansiedade e dos hábitos sociais, além do manejo das drogas de uso contínuo, como as medicações de ação cardiovascular, hipoglicemiantes, antiplaquetários, anticoagulantes, drogas modificadoras de doença reumática, agentes biológicos e antidepressivos. Conclusão: a APA tem como finalidade estratificar o risco anestésico e otimizar condições clínicas desfavoráveis, além de propor medidas de cuidados pré-operatórios que ofereçam segurança ao paciente.

Palavras-chave: Anestesia. Cuidados pré-operatórios. Período pré-operatório.

\section{ABSTRACT}

Introduction: The preanesthetic evaluation (PAE) is an indispensable practice in preoperative care for offering the condition to plan the anesthesia act and the surgery, maintaining the patient's physical and emotional safety. Objective: To present a review study on the most recent preanesthetic instructions available in the current literature. Methodology: Literature review study carried out at the following domains: PubMed, SciELO, British Journal of Anesthesia (BJA), Anesthesiology and Current Opinion. Result: The main guidelines address the stratification of the anesthetic-surgical risk, preoperative fasting, anxiety control and social habits, likewise the management of chronic usage drugs, such as the action of cardiovascular agents, antiplatelet drugs, hypoglycemic agents, anticoagulant, disease-modifying antirheumatic drugs, biological agents and antidepressants. Conclusion: PAE purpose is to stratify the anesthetic risk and optimize the unfavorable clinical conditions, likewise to propose ways for preoperative care that offer safety to the patient.

Keywords: Anesthesia. Preoperative care. Preoperative period.

Autor correspondente: Josiani dos Santos Garcez, Avenida Ministro José Américo, 150, Cambeba, Fortaleza, Ceará. CEP: 60824-245. Telefone: +55 85 98857-1607. E-mail: josiani_garcez@yahoo.com.br

Conflito de interesses: Não há qualquer conflito de interesses por parte de qualquer um dos autores.

Recebido em: 11 Mai 2018; Revisado em: 10 Jul 2018; Aceito em: 26 Ago 2018. 


\section{INTRODUÇÃO}

A avaliação pré-anestésica (APA) é uma consulta médica que deve ser realizada antes de qualquer anestesia, mesmo em cirurgias de urgência. Os objetivos da APA são obter informações sobre a história clínica e as condições físicas e emocionais do paciente, firmar o consentimento livre e esclarecido específico para a anestesia, estabelecer uma boa relação médico-paciente, além de reduzir a morbimortalidade do ato anestésico-cirúrgico e os custos dos cuidados perioperatórios. ${ }^{1}$

$\mathrm{O}$ adequado preparo do paciente para a anestesia e cirurgia é fundamental para o sucesso do procedimento e o retorno precoce desses pacientes às atividades diárias.

O objetivo desse artigo é apresentar uma revisão das principais orientações pré-anestésicas disponíveis na literatura atual.

\section{MATERIAIS E MÉTODOS}

Trabalho de revisão bibliográfica narrativa de literatura realizada nas bases eletrônicas PubMed, SciELO, British Journal of Anaesthesia (BJA), Aneshesiology e Current Opinion com os descritores padronizados pelos descritores em ciências da saúde: anesthesia, perioperative period, hyperglycemia, preoperative fasting, preoperative smoking, oral anticoagulants, antiplatelet, rheumatic drugs, antidepressant, seguidos dos conectivos or e/ ou and nos anos 2013 a 2017. Analisados trabalhos em inglês ou português, em humanos, com textos completos de revisão e estudo clínico, sendo filtrados 3812 artigos.

A primeira etapa foi a realização da leitura dos títulos dos artigos, pois apesar dos descritores, houve a seleção de artigos que não abordavam o tema, além de textos duplicados, sendo selecionados 42 artigos para a segunda etapa.

Nessa fase foi realizada a leitura dos resumos, dos quais foram selecionados 12 artigos que compõem essa revisão, por abordarem os cuidados perioperatórios desde a fisiologia às recomendações atuais de cuidados gerais e específicos de drogas de uso de continuo.

\section{ESTRATIFICAÇÃO DE RISCO PRÉ-ANESTÉSICO}

A APA é o momento ideal para esclarecer os benefícios e os riscos inerentes ao procedimento anestésico/cirúrgico, reduzindo a ansiedade do paciente e dos familiares. ${ }^{2}$

Os eventos adversos podem estar relacionados ao tipo de procedimento diagnóstico/terapêutico, mas também às comorbidades do paciente. A ciência das possíveis complicações melhora a comunicação no pós-operatório, facilitando a compreensão das condutas médicas durante o período perioperatório. ${ }^{2}$

O principal índice de risco utilizado para cirurgias não cardíacas é o Índice de Risco Cardíaco Revisado (RCRI, sigla do inglês Revised Cardiac Risk Index). O cálculo do RCRI é baseado na presença dos seguintes fatores: operação intraperitoneal, intratorácica ou vascular suprainguinal; doença arterial coronariana, insuficiência cardíaca congestiva, doença cerebrovascular, diabetes com insulinoterapia e creatinina pré-operatória maior que $2 \mathrm{mg} / \mathrm{dL}$. A presença de dois ou mais fatores de risco se enquadra como alto risco de evento cardíaco adverso, ocorrência de infarto agudo do miocárdio, edema agudo de pulmão, bloqueio atrioventricular total e parada cardiorrespiratória. ${ }^{2}$

Porém, o RCRI tem pouca acurácia para cirurgia vascular, sendo preferencialmente utilizado o Vascular Study Group of New England Cardiac Risk Index (VAG - CRI). Entretanto, quando o objetivo é analisar o risco global, pode-se utilizar a Surgical Risk Calculator (www.riskcalculator.facs.org). ${ }^{2}$

Essas ferramentas de avaliação de risco devem ser aplicadas a cada paciente de forma individualizada. ${ }^{3}$

\section{JEJUM PRÉ-OPERATÓRIO}

O jejum pré-operatório adequado aumenta a segurança no manejo de via aérea, reduzindo o risco de regurgitação e aspiração pulmonar de conteúdo gástrico. Porém, o jejum prolongado pode ter efeitos adversos ao reduzir a sensibilidade insulínica, aumentando as complicações pós-operatórias. ${ }^{4}$

Um controle glicêmico, jejum adequado como na Tabela 1 e o uso de soluções de carboidratos orais reduzem a resistência à insulina, possibilitando um menor risco de hiperglicemia e maior retenção de proteínas, melhorando cicatrização de feridas, função imune e mantendo a força muscular, além de proporcionar bem-estar ao paciente. ${ }^{3}$

Tabela 1. Recomendação de jejum pré-operatório.*

\begin{tabular}{ll}
\hline Material ingerido & Tempo de jejum mínimo \\
\hline Alimentos fritos, gordurosos ou carne & $8 \mathrm{~h}$ \\
Refeição leve ou leite não humano & $6 \mathrm{~h}$ \\
Fórmula infantil & $6 \mathrm{~h}$ \\
Leite materno & $4 \mathrm{~h}$ \\
Líquidos claros** & $2 \mathrm{~h}$ \\
\hline
\end{tabular}

Fonte: Adaptado de: Apfelbaum JL, Agarkar M, Connis RT, Coté CJ, Nickinovich DG, Warner MA. ${ }^{4}$

*Recomendação para pacientes saudáveis em procedimentos eletivos. Aplica-se a todas as idades.

**Água, suco de frutas sem polpa, bebidas a base de carboidrato, chá claro e café preto, exceto álcool.

\section{ANSIEDADE}

O estresse emocional, no período pré-operatório, deve-se à preocupação com os possíveis danos decorrentes da cirurgia, dor pós-operatória, separação da família, dependência física e medo da morte, que ativa o eixo hipotálamo-hipófise-adrenal e estimula a liberação de cortisol, hormônio contra insulínico. ${ }^{3}$ 
A ansiedade pode influenciar a percepção da dor pós-operatória, necessitando de uma demanda maior de analgésico para o controle da dor e, assim, reduzir o nível de satisfação com o procedimento. ${ }^{3}$

Dessa forma, a orientação sobre o ato anestésico e os cuidados perioperatórios são meios de reduzir a ansiedade, por vezes, dispensando o uso de drogas ansiolíticas., ${ }^{3,5}$

\section{OTIMIZAÇÃO DE CONDIÇÕES DE SAÚDE PRÉ-OPERATÓRIA}

O abuso de álcool, pela Organização Mundial de Saúde (OMS), é definido como a ingestão de $36 \mathrm{~g}$ de etanol ou equivalente a 3 bebidas padrão ao dia. Decorrente dessa condição, os pacientes têm o risco aumentado de sangramento perioperatório e infecção de ferida. Para reduzir esse risco, é necessário um mínimo de abstinência de 4 semanas. $^{5}$

O tabagismo pode aumentar o risco de complicações, como a cicatrização deficiente, infecção de ferida e pulmonar. A abstinência de no mínimo 12 horas, possibilita o benefício da redução de monóxido de carbono, disponibilizando mais oxigênio tecidual. Quando a cessação do fumo é de pelo menos 2 dias, podem-se melhorar a função ciliar e depuração das secreções pulmonares. Porém, apenas a abstinência de 4 a 8 semanas reduz complicações pulmonares pós-operatórias e melhora a cicatrização de feridas 5,6

No Brasil, a obesidade é estimada em 30\% dos pacientes cirúrgicos, sendo relacionada a aumento de morbimortalidade cardiovascular e pulmonar, dificultando ventilação, reduzindo capacidade residual funcional, atelectasias e shunts pulmonares, além de dificuldade de intubação, principalmente se houver associação com distúrbios respiratórios do sono. Portanto, deve-se estimular a perda de peso antes da cirurgia. ${ }^{2,5}$

Anemia, estado nutricional, hipertensão, diabetes, asma e doença pulmonar obstrutiva crônica são condições que devem ser otimizadas antes da cirurgia, por reduzir complicações perioperatórias. ${ }^{5} \mathrm{~A}$ anemia sobrecarrega o sistema cardiovascular, intensificando isquemia miocárdica e insuficiência cardíaca, aumentando morbidade e mortalidade como fator de risco independente. ${ }^{2}$

O acompanhamento multidisciplinar dessas situações é necessário. $^{5}$

\section{RECOMENDAÇÕES SOBRE AS DROGAS DE USO CONTÍNUO}

\section{A. Drogas de ação cardiovascular}

A hipertensão arterial sistêmica é uma condição comum na população, que pode aumentar a morbimortalidade se não controlada. Durante procedimentos cirúrgicos, há alterações hemodinâmicas mais pronunciadas em hipertensos, que se não controlados serão mais evidenciados. Aimportância da manutenção dos anti-hipertensivos, aliado ao planejamento de controle de dor e ansiedade, minimizam o risco de complicações pós-operatórias. ${ }^{2}$
Pacientes hipertensos possuem um grau de disfunção autômica que pode precipitar maior ocorrência de hipotensão durante o período intraoperatório. Esse quadro é mais frequente em pacientes que utilizam os inibidores da enzima conversora da angiotensina (IECA) e os bloqueadores do receptor de angiotensina II (BRA) que parecem favorecer a esses episódios, porém, a literatura tende a mantê-los pelo risco de descompensação da pressão arterial, insuficiência cardíaca e aumento do risco cardiovascular, como observado na Tabela $2 .{ }^{2}$

Tabela 2. Manejo pré-operatório de drogas cardiovasculares.

\begin{tabular}{ll}
\hline Anti-hipertensivos & Tempo de suspensão \\
\hline IECA/BRA* & Não suspender** \\
Diuréticos & Na manhã da cirurgia \\
Beta-bloqueadores & Manter \\
Bloqueadores de canal de cálcio & Manter \\
Alfa 2 - agonista & Manter \\
Estatina & Manter \\
Fenofibratos & Suspender 1 dia antes \\
\hline
\end{tabular}

Fonte: Adaptado de: Gualandro DM, Yu PC, Carameli B, Marques AC, Calderaro D, Luciana S, et al. ${ }^{2}$

*IECA: Inibidores da enzima conversora da angiotensina; BRA: Bloqueadores de receptores de angiotensina.

**Controverso na literatura - risco de hipotensão.

Em relação ao uso de beta bloqueadores, todas as medicações de uso crônico devem ser mantidas e reintroduzidas o mais precoce possível. Não há recomendação para introdução dessa medicação, nem aumento de doses no perioperatório, a menos que haja um período suficiente para otimização clínica.

As estatinas também são drogas importantes para serem mantidas em pacientes em uso crônico, em situações de cirurgia vascular e pacientes com doenças associadas como diabetes, doença arterial coronariana, doença cerebrovascular, pois além de reduzir níveis de colesterol, diminuem a inflamação e estabilizam placas de aterosclerose. ${ }^{2}$

\section{B. Hipoglicemiantes}

O controle glicêmico perioperatório depende da duração da cirurgia, invasividade do procedimento cirúrgico, tipo de técnica anestésica, tempo esperado para retornar a ingestão oral e terapia antidiabética de rotina. A hiperglicemia está associada a aumento de morbimortalidade perioperatória por complicações microvasculares e infecção pós-operatória. ${ }^{3,7}$

No período perioperatório, induzido pelo estresse cirúrgico, há uma resposta neuroendócrina de liberação de hormônios contra reguladores, como glucagon, epinefrina e cortisol, que podem contribuir para o aumento da glicemia. Em pacientes insulinos dependentes, deve-se monitorizar a glicemia durante o jejum pré-operatório sem suspender a insulina basal pelo risco de cetoacidose diabética, enquanto no diabético tipo 2 aumenta o risco de estado hiperosmolar. ${ }^{7}$ 
O manejo da metformina, hipoglicemiante oral da classe das biguanidas, tem recomendação de manter o uso no dia da cirurgia para pacientes que passam por curto período de jejum com apenas uma refeição perdida, porém, aqueles que serão submetidos a procedimentos com uso de contraste intravenoso ou longo tempo cirúrgico, a metformina é interrompida ao iniciar o jejum e reiniciada após retomada a dieta normal. Se a função renal apresentar taxa de filtração glomerular menor que $45 \mathrm{ml} / \mathrm{min}$ no pré-operatório ou desenvolver disfunção no pós-operatório, essa droga é interrompida até normalização da função renal. ${ }^{7}$

Os diabéticos tipo 2 que utilizam insulina devem continuar a insulinoterapia, somente com redução de $25 \%$ da dose normal na noite anterior da dose basal de Glargina/Detemir ou na manhã da cirurgia se a dose for aplicada duas vezes ao dia. No caso da insulina Neutral Protamine Hagedorn (NPH) deve-se reduzir $20 \%$ da dose na noite anterior e $50 \%$ na manhã da cirurgia. Também recomenda-se manter a dose de NPH pela manhã em diabéticos tipo 2 e glicemia de jejum menor que $120 \mathrm{mg} / \mathrm{dL}$.

Diabéticos tipo 1 devem manter insulinoterapia para evitar hiperglicemia grave e cetoacidose. Devem receber $80 \%$ de dose basal na noite anterior à cirurgia e na manhã da cirurgia. A insulina pós-prandial é interrompida ao iniciar o jejum.

O manejo da insulina na Tabela 3 e hipoglicemiantes orais na Tabela 4 apresentam algumas divergências na literatura., ${ }^{2,7}$

Tabela 3. Manejo das insulinas pré-operatórias.

\begin{tabular}{llll}
\hline Insulinas & \multicolumn{2}{c}{ Dia antes da cirurgia } & No dia da cirurgia \\
\cline { 2 - 4 } & Manhã & Noite & Manhã \\
\cline { 2 - 4 } NPH * & $80 \%$ dose & $80 \%$ dose & $50 \%$ dose ** \\
Determir, Glargina & Dose usual & $80 \%$ dose & $80 \%$ dose *** \\
Rápida ou Ultrarrápida & Dose usual & Dose usual & Suspender \\
\hline
\end{tabular}

Fonte: Adaptado de: Duggan EW, Carlson K, Umpierrez GE.?

*NPH: Neutral Protamine Hagedorn

**Se glicemia sanguínea $\geq 120 \mathrm{mg} / \mathrm{dl}$. Suspender se glicemia $<120 \mathrm{mg} / \mathrm{dl}$.

Manter glicemia $4 / 4 \mathrm{~h}$ e, se necessário, glicose $5 \mathrm{~g} / \mathrm{h}$ conforme glicemia.

***Se paciente utilizar terapia basal pela manhã ou duas vezes ao dia.

Manter glicemia $4 / 4 \mathrm{~h}$ e, se necessário, glicose $5 \mathrm{~g} / \mathrm{h}$ conforme glicemia.

Tabela 4. Manejo dos hipoglicemiantes orais.

\begin{tabular}{lll}
\hline Classes & Medicamentos & Tempo de suspensão \\
\hline Biguanidas & Metformina & No dia da cirurgia* \\
Sulfoniuréias & Glicazida & No dia da cirurgia \\
& Glibenclamida & \\
& Glimepirida & \\
Tiazolidinedionas & Pioglitazona & Manter no dia da cirurgia** \\
Acarbose & Acarbose & Suspender 1 dia antes \\
Glinidas & Repaglinida & No dia da cirurgia \\
Inibidores DPP-4 & Sitagliptina & Manter no dia da cirurgia \\
Agonistas do GLP-1 & Exenatida & No dia da cirurgia \\
Inibidores SLGT-2 & Dapaglifozina & Suspender 1 dia antes*** \\
\hline
\end{tabular}

Fonte: Adaptado de: Duggan EW, Carlson K, Umpierrez GE.?

*Suspender ao iniciar o jejum se procedimento com administração de contraste e cirurgia longa.

Suspender em pacientes com taxa de filtração glomerular $<45 \mathrm{ml} / \mathrm{min}$ a qualquer momento do pré-operatório. Porém, pode ser mantida no dia da cirurgia se ingestão oral normal (apenas uma refeição perdida) no dia do procedimento e cirurgia minimamente invasiva.

**Suspender no dia da cirurgia se previsão de redução de ingestão oral no pós-operatório ou cirurgia extensa com variação hemodinâmica.

****isco de Cetoacidose diabética

DPP-4: dipeptidil peptidase-4

GLP-1: peptídeo semelhante a glucagon 1

SLGT-2: transportador sódio-glicose do tipo 2 


\section{Antiplaquetários}

Os antiplaquetários são drogas essenciais no cuidado do paciente com risco de trombose cardiovascular. $\mathrm{O}$ uso mais comum inclui a prevenção secundária de acidente vascular cerebral e infarto agudo do miocárdio. ${ }^{8}$

O manejo dessas drogas no pré-operatório depende da indicação clínica do medicamento e risco de sangramento perioperatório observado na Tabela 5. Quanto maior o risco de sangramento, maior a necessidade de uma indicação precisa do uso do antiplaquetário, vide Tabela $6 .{ }^{8,9}$

Quando há indicação de dupla agregação plaquetária e risco elevado de trombose de stent pós-angioplastia, insuficiência coronariana aguda, podem ser consideradas para uma terapia de ponte com antiagregante parenteral, como o inibidor de gligoproteina IIb/IIIa.

Tabela 5. Risco de complicação por sangramento pelo procedimento cirúrgico.

\begin{tabular}{lll}
\hline Alto risco & Risco Moderado & Baixo risco \\
\hline Neurocirurgia & Cirurgia visceral & Cirurgia plástica \\
Cirurgia câmara posterior olho & Cirurgia cardiovascular & Cirurgia ortopédica menor \\
& Cirurgia ortopédica maior & Cirurgia orelha, nariz, garganta \\
& Reconstrução orelha, nariz ou garganta & Cirurgia câmara anterior do olho \\
& Cirurgia urológica & \\
\hline
\end{tabular}

Fonte: Adaptado de: Koenig-Oberhuber V, Filipovic M; ${ }^{8}$ Oprea AD, Popescu WM. ${ }^{9}$

Tabela 6. Manejo pré-operatório de antiplaquetários.

\begin{tabular}{llll}
\hline Antiplaquetários & \multicolumn{2}{c}{ Tempo de suspensão conforme risco de sangramento } \\
\hline & Alto risco & Risco moderado & Baixo risco \\
\hline Aspirina & Profilaxia primária: suspender 7 dias & Profilaxia primária: suspender 7 dias & Manter \\
& $\begin{array}{l}\text { Profilaxia secundária: } \\
\text { máximo de } 7 \text { dias }\end{array}$ & & \\
Clopidogrel & 7 dias & 7 dias & Manter* \\
Prasugrel & $7-10$ dias & Profilaxia secundária: Manter & Manter* \\
Ticagrelol & 5 dias & $7-10$ dias & Manter* \\
Cilostazol & 2 dias & 5 dias & Manter \\
Abciximab & $2-5$ dias & Manter & $2-5$ dias \\
Eptifibatide & $8-24 \mathrm{~h}$ & $2-5$ dias & $8-24 \mathrm{~h}$ \\
Tirofiban & $8-24 \mathrm{~h}$ & $8-24 \mathrm{~h}$ & $8-24 \mathrm{~h}$ \\
Naproxeno & 3 dias & $8-24 \mathrm{~h}$ & Manter \\
Ibuprofeno & 10 horas & 3 dias & Manter \\
Diclofenaco & 10 horas & 10 horas & Manter \\
Indometacina & 1 dia & 10 horas & Manter \\
Inibidor COX-2** & Manter & 1 dia & Manter \\
\hline
\end{tabular}

Fonte: Adaptado de: Koenig-Oberhuber V, Filipovic M; ${ }^{8}$ Oprea AD, Popescu WM. ${ }^{9}$

*Controverso: avaliar técnica anestésica

**COX-2: cicloxigenase-2

\section{Anticoagulantes}

Os anticoagulantes são medicações utilizadas tanto para prevenção como tratamento de doenças tromboembólicas como fibrilação atrial, prótese valvar metálica e tromboembolismo pulmonar. $^{10}$
Deve-se avaliar a relação risco-benefício do uso de anticoagulantes, considerando-se a indicação do uso e os fatores cirúrgicos para decidir sobre a suspensão da droga.

Nos casos que o risco tromboembólico seja elevado e tempo de suspensão da droga prolongado, deve-se realizar terapia de 
ponte com heparina quando a Razão Normalizada Internacional, do inglês International normalized ratio (INR), for menor que 2. A suspensão da heparina não fracionada deve ocorrer 4-6 horas antes do procedimento, e se heparina de baixo peso molecular, 24 horas antes. No pós-operatório, reintroduzir a heparina pelo menos 24 horas após o procedimento cirúrgico, porém, se houver alto risco de sangramento deve-se atrasar a reintrodução para 48-72 horas após a cirurgia, concomitante o início da varfarina, para somente após a INR na faixa terapêutica, suspender o uso da heparina. ${ }^{2,10}$

A varfarina é um anticoagulante que tem influência de fatores como idade, função renal e interferência de outras medicações, sendo, portanto, necessária avaliação laboratorial do INR no dia anterior à cirurgia para assegurar um valor $<1,5$ ou ter tempo para reversão com vitamina K e reavaliação no INR no dia seguinte. ${ }^{2,10}$

A dabigatran é um anticoagulante inibidor direto da trombina, bloqueando a conversão do fibrinogênio em fibrina de modo reversível. Usada para prevenção de acidente vascular cerebral, fibrilação atrial não valvar e tratamento de tromboembolismo venoso em cirurgias ortopédicas. Apresenta início de ação rápido de 30 minutos e pico de concentração de 120 minutos, além de meia vida curta de 12-17 horas, porém aumentada em insuficiência renal.

A rivaroxabana age inibindo o fator Xa, bloqueando a conversão de protrombina em trombina. Tem início de ação rápido e pico de concentração de 2-4 horas, meia-vida de 5-9 horas em jovens e 11-13 horas em idosos, também influenciado pela função renal.

O gerenciamento perioperatório dos novos anticoagulantes é variável e ainda com evidência fraca, utilizando 5 meiasvidas para suspensão conforme a Tabela $7 .{ }^{10}$

\section{E. Drogas modificadoras de doença reumática/agentes biológicos}

As doenças reumatológicas são prevalentes no cenário de saúde, porém, as medicações são consideradas complexas e desconhecidas por muitos profissionais, o que dificulta a gestão desses pacientes na sala cirúrgica. ${ }^{11}$ Eles estão expostos a maior risco de infecção, impactando nas complicações perioperatórias. ${ }^{11}$

O metotrexate deve ser continuado durante o perioperatório sem aumentar risco de infecção e comprometimento da cicatrização, porém, em pacientes com doença renal crônica e diabetes mellitus não controlado, deve-se suspender por uma semana, pois já podem aumentar esses riscos de maneira independente. $^{11}$

A hidroxicloroquina em pacientes lúpicos reduz risco cardiovascular, resistência a insulina, eventos tromboembólicos, infecção e mortalidade ao controlar a doença de base, portanto, deve ser mantida no perioperatório.

A Tabela 8 apresenta o tempo de suspensão e retorno das drogas modificadoras de doença reumática.

Em relação aos agentes biológicos, para procedimentos menores não há necessidade de interromper, pois não aumenta risco de infecção, porém, em cirurgias de grande porte deve-se suspender duas meias-vidas e retomar de 10-14 dias após a cirurgia, observar Tabela 9.

O rituximabe é um anticorpo monoclonal anti CD-20 que depleta linfócitos B e pode produzir hipogamaglobinemia grave, devendo ser avaliado os níveis séricos de IgG no pré-operatório.

Tabela 7. Manejo pré-operatório de anticoagulantes.

\begin{tabular}{lll}
\hline Mecanismo ação & Medicação & Tempo suspensão \\
\hline Inibe fatores vitamina K dependente (II, VII, IX, X) & Varfarina sódica & 5 dias e INR* $\leq 1,4$ \\
Inibe IIa, Xa, IXa & HNF** intravenoso & 4 horas \\
Inibe IIa, Xa, IXa & HNF** subcutânea 2-3 vezes ao dia & $8-10$ horas \\
Inibe IIa, Xa, IXa & HBPM*** profilática $^{*}$ & 12 horas \\
Inibe IIa, Xa, IXa & HBPM***terapêutica $^{*}$ & 24 horas \\
Inibe fator Xa & Fondaparinux & 4 dias \\
Inibe fator IIa & Dabigatran (300mg/dia) & $4-5$ dias $^{+}$ \\
Inibe fator Xa & Rivaroxaban $(20 \mathrm{mg} /$ dia) & 3 dias $^{++}$ \\
Inibe fator Xa & Apixaban (10mg/dia) & $3-4$ dias $^{+++}$ \\
Agentes fibrinolíticos & Estreptoquinase & $48 \mathrm{~h}$ \\
\end{tabular}

Fonte: Adaptado de: Koenig-Oberhuber V, Filipovic M; ${ }^{8}$ Breuer G, Weiss DR, Ringwald J. ${ }^{10}$

*INR: International normalized ratio

**HNF: Heparina não fracionada.

***HBPM: Heparina de baixo peso molecular.

$+\mathrm{Se} \mathrm{ClCr} \leq 50 \mathrm{ml} / \mathrm{min}$ e idade $>75$ anos, suspender 6 dias.

$++\mathrm{Se} \mathrm{ClCr} \leq 30 \mathrm{ml} / \mathrm{min}$, suspender 4 dias.

$+++\mathrm{Se} \mathrm{ClCr} \leq 30 \mathrm{ml} / \mathrm{min}$, suspender medicação por 5 dias. 
Tabela 8. Manejo pré-operatório das drogas anti-reumáticas modificadoras de doença.

\begin{tabular}{ll}
\hline Medicação DMARDs* & Tempo suspensão \\
\hline Metotrexate & Manter** \\
Hidroxicloroquina & Manter \\
Sulfasalazina & Manter \\
Leflunomide & 2 semanas, retomar 3 dias após cirurgia *** \\
Ciclosporina & 1 semana, retomar 1 semana após cirurgia \\
Azatioprina & Manter \\
Micofenolato mofetil & 1 semana, retomar $1-2$ semanas após cirurgia \\
\hline
\end{tabular}

Fonte: Adaptado de: Franco AS, Luamoto LR, Pereira RM. ${ }^{11}$

*DMARDs: Drogas anti-reumáticas modificadoras de doença, sigla do inglês Disease modifying antirheumatic drugs. **Se paciente com doença renal crônica, diabetes mellitus descompensada, deve-se suspender Metotrexate por 1 semana.

**** Controverso.

Tabela 9. Manejo pré-operatório dos agentes biológicos.

\begin{tabular}{lll}
\hline Agentes biológicos & Mecanismo de ação & Tempo suspensão \\
\hline Etanercept & Anti - TNF* & 10 dias \\
Golimumab & Anti - TNF & 28 dias \\
Adalimumab & Anti - TNF & 30 dias \\
Infliximab & Anti - TNF & 19 dias \\
Certolizumab & Anti - TNF & 28 dias \\
Rituximab & Inibidor de células B & 100 dias \\
Abatacept & Inibidor de células T & Intravenoso -4 semanas \\
& & Subcutâneo -2 semanas \\
Tocilizumab & Antagonista receptor IL-6** & 26 dias \\
Anakinra & Antagonista receptor IL-1 & $1-2$ dias \\
\hline
\end{tabular}

Fonte: Adaptado de: Franco AS, Luamoto LR, Pereira RM. ${ }^{11}$

*TNF: Fator de necrose tumoral, sigla do inglês tumor necrosis fator.

**IL: Interleucina.

\section{F. Antidepressivos}

Em geral, os antidepressivos são continuados no período perioperatório para evitar síndrome de abstinência e piora da depressão. ${ }^{12}$

Os antidepressivos tricíclicos podem aumentar o risco de arritmias quando usados em conjunto com alguns anestésicos. ${ }^{12}$

Os inibidores da recaptação da serotonina estão associados a aumento do risco de sangramento por efeito secundário na agregação plaquetária. ${ }^{13}$

Os inibidores de monoamino oxidase (IMAO) apresentam várias interações com medicações anestésicas como tramadol e meperidina, aumentando a liberação de serotonina. $\mathrm{O}$ uso de drogas simpaticomiméticos indiretas associado aos IMAO pode liberar uma grande quantidade de noradrenalina e produzir uma crise hipertensiva. ${ }^{12}$

Como as interações medicamentosas são previsíveis, os antidepressivos são seguros para manter no perioperatório, observando-se os cuidados com o manejo das drogas anestésicas. $^{12}$

\section{CONCLUSÃO}

A avaliação pré-anestésica tem como finalidade não somente estratificar o risco do ato anestésico e cirúrgico, mas propor medidas antecipadas que podem oferecer benefício ao paciente na situação específica do procedimento.

Essas orientações são baseadas na literatura recente e os tópicos controversos devem ser individualizados. 


\section{REFERÊNCIAS}

1. Brasil. Resolução CFM N 1.802/2006, de 01 novembro 2006. Dispõe sobre a prática do ato anestésico [Internet]. Brasília: Conselho Federal de Medicina; 2006 [acesso em: 20 jan 2018]. Disponível em: http://www.portalmedico.org.br/resolucoes/cfm/2006/1802_2006. $\mathrm{htm}$

2. Gualandro DM, Yu PC, Carameli B, Marques AC, Calderaro $\mathrm{D}$, Luciana $\mathrm{S}$, et al. $3^{\mathrm{a}}$ Diretriz de Avaliação Cardiovascular Perioperatória da Sociedade Brasileira de Cardiologia. Arq Bras Cardiol. 2017; 109(3Supl.1):1-104.

3. Scott MJ, Balbine G, Fearon KC, Feldheiser A, Feldman LS, Gan TJ, et al. Enhanced Recovery After Surgery (ERAS) for gastrointestinal surgery, part 1: pathophysiological considerations. Acta Anaesthesiol Scand. 2015;59(10):1212-31.

4. Apfelbaum JL, Agarkar M, Connis RT, Coté CJ, Nickinovich DG, Warner MA. Practice guidelines for preoperative fasting and the use of pharmacologic agents to reduce the risk of pulmonary aspiration: application to healthy patients undergoing elective procedures. Anesthesiology. 2017; 126(3):376-93.

5. Feldheiser A, Aziz O, Balbini G et al. Enhanced Recovery After Surgery (ERAS) for gastrointestinal surgery, Part 2: consensus statement for anaesthesia practice. Acta Anaesthesiologica Scandinavica. 2016; 60:289-334.

6. Gronkjaer M, Eliasen M, Skov-Ettrup LS, Tolstrup JS, Christiansen AH, Mikkelsen SS, et al. Preoperative smoking status and postoperative complications: a systematic review and metaanalysis. Ann Surg. 2014;259(1):52-71.

7. Duggan EW, Carlson K, Umpierrez GE. Perioperative hyperglycemia management. Anesthesiology. 2017;126(3):547-60.

8. Koenig-Oberhuber V, Filipovic M. New antiplatelet drugs and new oral anticoagulants. Br J Anaesth. 2016;117(Supl. 2):74-84.

9. Oprea AD, Popescu WM. Perioperative management of antiplatelet therapy. Br J Anaesth. 2013;111(Supl 1):3-17.

10. Breuer G, Weiss DR, Ringwald J. 'New' direct oral anticoagulants in the perioperative setting. Curr Opin Anesthesiol. 2014;27(4):40919.

11. Franco AS, Luamoto LR, Pereira RM. Perioperative management of drugs commonly used in patients with rheumatic diseases: a review. Clinics (São Paulo). 2017;72(6):386-90.

12. Golembiewski J, Pharm D. Antidepressant pharmacology and perioperative implications. J Post Anesth Nurs. 2014;29(4):327-329.

13. Mahdanian AA, Rej S, Bacon SL, Ozdin D, Lavoie KL, Looper K. Serotonergic antidepressants and perioperative bleeding risk: a systematic review. Expert Opin. Drug Saf. 2014;13(6):695-704.

\section{Como citar:}

Garcez JS, Sousa LC, Novais MB Neta, Maia FL, Araújo FP. Principais recomendações em cuidados pré-operatórios. Rev Med UFC. 2019 janmar;59(1):53-60. 